\title{
Connectivity, Traceability and Hamiltonicity
}

\author{
P. Mafuta ${ }^{1}$. J. P. Mazorodze ${ }^{2} \cdot$ S. Munyira ${ }^{2}$. J. Mushanyu ${ }^{2}$
}

Received: 14 April 2021 / Revised: 10 September 2021 / Accepted: 15 November 2021 /

Published online: 10 January 2022

(c) The Author(s), under exclusive licence to Springer Science+Business Media LLC, part of Springer Nature 2022

\section{Abstract}

Let $G$ be a simple, connected, triangle-free graph with minimum degree $\delta$, order $n$ and leaf number $L(G)$. If $G$ has a cut-vertex, we prove that $L(G) \geq 4 \delta-4$ and $n \geq 4 \delta-1$. Both lower bounds are sharp. The lower bound on the leaf number strengthens a result by Mukwembi for triangle-free graphs. As corollaries, we deduce sufficient conditions for connectivity, traceability and Hamiltonicity in triangle-free graphs. As an easy extension of a result by Goodman and Hedetiniemi, we show that a simple, connected, claw-free, paw-free graph $G$ is Hamiltonian if and only if $G$ is not a path. We consider only simple graphs, that is, graphs with neither loops nor multiple edges.

Keywords Leaf number $\cdot$ Minimum degree $\cdot$ Order $\cdot$ Connectivity $\cdot$ Cycles $\cdot$ Paths

Mathematics Subject Classification $05 \mathrm{C} 38 \cdot 05 \mathrm{C} 40 \cdot 05 \mathrm{C} 45$

\section{Introduction}

Let $G=(V, E)$ be a connected graph with vertex set $V(G)$ and edge set $E(G)$. The connectivity $\kappa(G)$ of $G$ refers to the minimum number of vertices whose deletion

P. Mafuta

phillipmafuta@gmail.com

J. P. Mazorodze

mazorodzejaya@gmail.com

S. Munyira

munyirask@gmail.com

J. Mushanyu

mushanyuj@gmail.com

1 Department of Mathematics and Applied Mathematics IB74, University of the Free State,

Bloemfontein, South Africa

2 Department of Mathematics and Computational Sciences, University of Zimbabwe, Harare,

Zimbabwe 
disconnects $G$. $G$ is said to be $k$-connected if $\kappa(G) \geq k$. A vertex $u \in V(G)$ is called a cut-vertex or articulation vertex if the deletion of $u$ disconnects $G$. It follows that every 2-connected graph has no cut-vertex. The degree of a vertex $y \in V(G)$, denoted $\operatorname{deg}_{G}(y)$, refers to the number of edges in $G$ incident with $y$. The smallest of the degrees of vertices in $G$ is called the minimum degree, denoted $\delta=\delta(G)$. A leaf is a vertex of degree one. A tree refers to a connected graph without cycles. The leaf number $L(G)$, whose applications in network designs are pertinent, is the maximum number of leaf vertices contained in a spanning tree of $G$. We denote by $C_{n}$, a cycle of length $n$. A triangle is a $C_{3}$ graph. $G$ is called a $C_{n}$-free if it has no cycle of length $n$. Thus, $G$ is triangle-free if it is a $C_{3}$-free graph. A cycle $C$ is said to be dominating if no pair of vertices outside $C$ are adjacent. A claw is a $K_{1,3}$ bipartite graph. Let $K_{1,3}=\left\{x x_{1}, x x_{2}, x x_{3}\right\}$. Then the addition of just one of the edges $x_{1} x_{2}, x_{1} x_{3}$ and $x_{2} x_{3}$ yields a graph called a paw, see also $[6,9,10]$ for more details on claws and paws.

Let $H$ be a graph. Then, $H$ is a subgraph of $G$ if $E(H) \subseteq E(G)$ and $V(H) \subseteq V(G)$. The graph $H$ is a spanning subgraph of $G$, if $V(H)=V(G)$. A subgraph, $G[S]$ of $G$, whose vertex set is $S$ is induced if its edge set consists of all edges in $E(G)$ which have all their endpoints in $S$. If $H \neq C_{n}$, then $G$ is $H$-free if it has no induced subgraph isomorphic to $H$. Equivalently, $H$ is called a forbidden subgraph of $G$. The graph $G$ is Hamiltonian if it contains a spanning cycle and traceable if it has a spanning path. Thus, every Hamiltonian graph is traceable. For vertex disjoint graphs $G_{1}, G_{2}, \ldots, G_{k}$, the sequential join $G_{1}+G_{2}+\ldots+G_{k}$ is the graph obtained from the union of $G_{1}, G_{2}, \ldots, G_{k}$ by joining every vertex of $G_{i}$ to every vertex of $G_{i+1}$ for $i=1,2, \ldots, k-1$.

Existence of spanning paths and cycles in graphs helps to solve data communication problems among other applications (see for instance [7,20]). The problem of determining the leaf number and whether spanning paths or cycles exists in a graph is N P-complete (see for instance [8]). Sufficient conditions for a graph to be Hamiltonian has a long history $[4,5,18,19]$. The search for sufficient conditions for a graph to be Hamiltonian remains an on going research area (see for instance $[12,16,17]$ ). It is known that for a graph to be Hamiltonian necessarily, it must be 2-connected. Hence, it becomes natural to ask for conditions that guarantee a graph to be 2-connected. For graphs in general, Mukwembi [16] established a sufficient condition for a graph to be 2 -connected based on leaf number and minimum degree. Precisely, he proved the following result:

Theorem 1 [16] Let $G$ be a connected graph with minimum degree $\delta$ and leaf number $L(G)$. If $G$ has a cut-vertex, then $L(G) \geq 2 \delta$. Moreover, the bound is tight.

Theorem 1 was shown to be best in the sense that, for each $\delta$, the graph $G_{2 \delta+1}=$ $K_{\delta}+K_{1}+K_{\delta}$ contains a cut-vertex and has leaf number $L\left(G_{2 \delta+1}\right)=2 \delta$. For graphs in general, Theorem 1 has been used as a powerful tool in the establishment of sufficient conditions for traceability or Hamiltonicity based on leaf number and minimum degree (see for instance [11-14,16]). This motivated us to establish a similar bound for triangle-free graphs with the hope that the results will in the near future play an important role in providing sufficient conditions for triangle-free graphs to be either Hamiltonian or traceable. In fact, some of the corollaries and theorems in this paper demonstrate how useful the main results are in providing sufficient conditions for 
Hamiltonicity or traceability in triangle-free graphs. Thus, in this paper, we strengthen Theorem 1 for triangle-free graphs.

Apart from this, we establish a similar lower bound on the order in terms of minimum degree. In addition, we show that the results are sharp. Further, we deduce sufficient conditions for a triangle-free graph to be 2-connected, traceable or Hamiltonian. We mention here that similar ideas used to prove results in this paper can be used to provide similar results for graphs of given girth.

The study of path- and cycle-related properties in triangle-free graphs has been dealt with and is still an ongoing research area (see for instance [1-3,15]). The next theorem is crucial in settling some of the corollaries in this paper.

Theorem 2 [2] Let $G$ be a 2-connected, triangle-free graph of order $n$ and minimum degree $\delta$. If $C$ is a longest cycle in $G$, then $|V(C)| \geq \min \{4 \delta-4, n\}$ or $C$ is a dominating cycle.

Goodman and Hedetineimi [9] proved the following result:

Theorem 3 Let $G$ be a 2-connected, claw-free, paw-free graph. Then $G$ is Hamiltonian.

Following Theorem 3, we note that it has gone unnoticed that the only non-Hamiltonian claw-free, paw-free graph is a path $P_{n}$. One of the results in this paper proves that every connected claw-free, paw-free graph $G$ is Hamiltonian unless $G=P_{n}$. This improves Theorem 3 for claw-free, paw-free graphs.

Apart from notation already defined, we use the following: For vertices $u, v \in$ $V(G)$, the distance, $d_{G}(u, v)$ is the length of a shortest path between $u$ and $v$ in $G$. The neighbourhood or closed-neighbourhood, $N_{G}[v]$, of a vertex $v$ in $G$ is given by

$$
N_{G}[v]=\left\{u \in V(G): d_{G}(u, v) \leq 1\right\} .
$$

The open-neighbourhood, $N_{G}(v)$, of a vertex $v$ in $G$ is given by

$$
N_{G}(v)=\left\{u \in V(G): d_{G}(u, v)=1\right\} .
$$

Thus, $N_{G}[v]=\{v\} \cup N_{G}(v)$. The $k$ th neighbourhood of $v, N_{G}^{k}[v]$, is defined as

$$
N_{G}^{k}[v]=\left\{u \in V(G): d_{G}(u, v) \leq k\right\} .
$$

Here, the $k$ th open-neighbourhood of $v, N_{G}^{k}(v)$, is defined as

$$
N_{G}^{k}(v)=\left\{u \in V(G): d_{G}(u, v)=k\right\} .
$$

Further, $G \backslash\{v\}$ denotes the graph obtained from $G$ by deleting a vertex $v$. If $H$ is a subgraph of $G$, we denote by $N_{H}^{k}(v)$ the $k$ th open-neighbourhood of $v$ in $H$. Where there is no ambiguity, we drop the argument $G$. 


\section{Main Results}

We now prove the main result of this paper and we deduce from it sufficient conditions for a triangle-free graph to be 2-connected. The following result strengthens Theorem 1 for triangle-free graphs.

Theorem 4 Let $G$ be a connected, triangle-free graph with minimum degree $\delta$, order $n$ and leaf number $L(G)$. If $G$ has a cut-vertex, then $L(G) \geq 4 \delta-4$ and $n \geq 4 \delta-1$. Moreover, the bounds are sharp.

Proof The proof is trivial for $\delta=1$. For $\delta=2$, the bound on the leaf number follows by an application of Theorem 1 . To establish the bound on the order, assume to the contrary that $n \leq 6$. Since $\delta=2, G$ has a cycle. Let $C_{k}$ be a longest cycle in $G$. Then $4 \leq k \leq 6$, since $G$ is a triangle-free graph and $n \leq 6$. If $k=5$ or 6 , then $G$ is 2-connected, since there is at most one vertex outside $C_{k}$ and $\delta=2$, a contradiction. Similar arguments holds if $k=4$ and $n \leq 5$. So, it is adequate to consider $k=4$ and $n=6$.

In this case, let $V\left(C_{4}\right)=\left\{v_{1}, v_{2}, v_{3}, v_{4}\right\}, E\left(C_{4}\right)=\left\{v_{1} v_{2}, v_{2} v_{3}, v_{3} v_{4}, v_{4} v_{1}\right\}$ and, $u_{1}, u_{2} \notin V\left(C_{4}\right)$. Assume first that $u_{1} u_{2} \in E(G)$. Since $\delta=2$, apart from $u_{2}$, we may assume that $u_{1}$ is adjacent $v_{1}$. Similarly, $u_{2}$ has a neighbour on $C_{4}$ which is not $v_{1}$, since $G$ is a triangle-free graph. Thus, the second neighbour of $u_{2}$ is in the set $\left\{v_{2}, v_{3}, v_{4}\right\}$. But this yields a cycle of length at least 5 , a contradiction. So this case fails.

Assume that $u_{1} u_{2} \notin E(G)$. Then each $u_{i}$ has at least two neighbours on $C_{4}$ which are not consecutive. We may assume that $u_{1} v_{1}, u_{1} v_{3} \in E(G)$. Then, if we join $u_{2}$ to at least two of its neighbours in $V\left(C_{4}\right)$ we obtain a 2-connected graph, which is not allowed. In either case the supposition that $n \leq 6$ yields a contradiction. Thus, we conclude that $n \geq 7$ as desired.

Consider $\delta \geq 3$. Let $u$ be a cut-vertex of $G$. Further, let $x$ and $y$ be neighbours of $u$ that are in different components of $G \backslash\{u\}$. In addition, let $G_{1}$ and $G_{2}$ be the components of $G \backslash\{u\}$ containing $x$ and $y$, respectively. By our choice of $u, x$ and $y$, there following property holds:

Property 1 Let $x$ and $y$ be as defined above. Then $N_{G}(x) \cap N_{G}(y)=\{u\}$ and for all $s, k ; s \geq 1, k \geq 2$, we have $N_{G \backslash\{u\}}^{s}(x) \cap N_{G \backslash\{u\}}^{k}(y)=N_{G \backslash\{u\}}^{k}(x) \cap N_{G \backslash\{u\}}^{s}(y)=$ $\emptyset$.

Let $x_{1}, x_{2}, \ldots, x_{\delta-1} \in N(x) \backslash\{u\}$ and $y_{1}, y_{2}, \ldots, y_{\delta-1} \in N(y) \backslash\{u\}$. By Property 1 , elements of $N(x) \backslash\{u\}$ are different from those in $N(y) \backslash\{u\}$. By the same argument for all $i, j, x_{i}$ cannot be adjacent to $y_{j}$. Also, since $G$ is triangle-free, for $i \neq j, x_{i}$ cannot be adjacent to $x_{j}$ and $y_{i}$ cannot be adjacent to $y_{j}$. Following these arguments, let $w_{1}, w_{2}, \ldots, w_{\delta-1}$ and $z_{1}, z_{2}, \ldots, z_{\delta-1}$ be neighbours of $x_{1}$ and $y_{1}$, respectively, apart from $x$ and $y$. Then $G$ has a tree $T$ shown in Fig. 1, with $4 \delta-6$ leaves and order $4 \delta-1$, where 


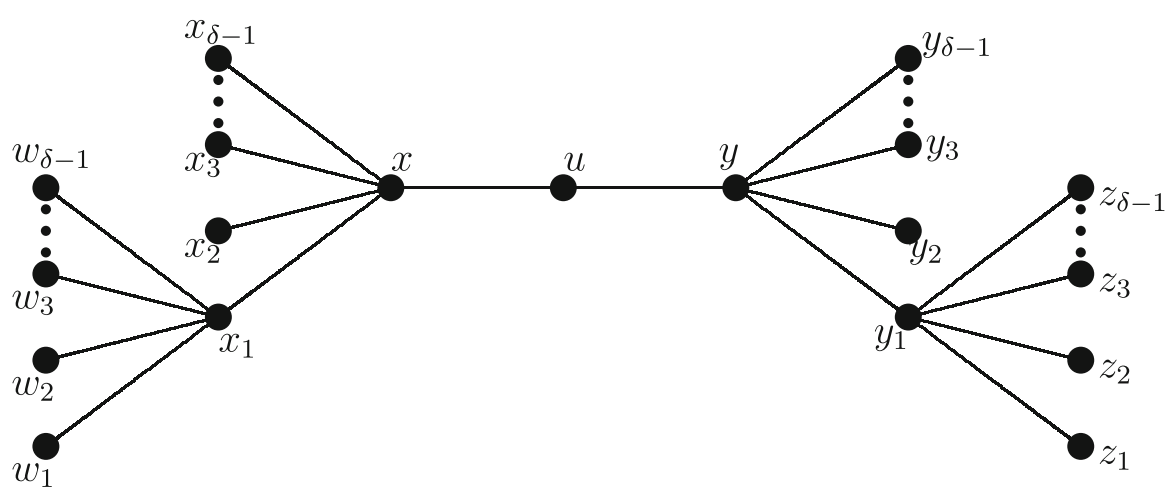

Fig. 1 The tree $T$

$$
V(T)=\left\{u, x, y, w_{1}, w_{2}, \ldots, w_{\delta-1}, x_{1}, x_{2}, \ldots, x_{\delta-1}, y_{1}, y_{2}, \ldots, y_{\delta-1}, z_{1}, z_{2}, \ldots, z_{\delta-1}\right\}
$$

and

$$
E(T)=\left\{u x, u y, x x_{i}, y y_{i}, x_{1} w_{i}, y_{1} z_{i} 1 \leq i \leq \delta-1\right\}
$$

Thus,

$$
n \geq|V(T)|=4 \delta-1 .
$$

To settle the bound on the leaf number, we prove the following lemmas using the tree $T$ defined above.

Lemma 1 Assuming the above notation, if u has at least one neighbour outside $T$, then $G$ has a tree with at least $4 \delta-4$ leaves.

Proof of Lemma 1 If $u$ has at least two neighbours outside $T$ then we are done. So, we can assume that $u$ has only one neighbour, say, $z$ outside $T$. Let $T_{1}=T \cup\{u z\}$. Then $T_{1}$ has $4 \delta-5$ leaves. We can also assume that no vertex in $G$ has at least two neighbours outside $T_{1}$, otherwise, we get the required tree and we are done. Hence, $z$ has at least two neighbours in $T$, which are neither $x$ nor $y$, otherwise, we obtain a triangle, which is not allowed.

By Property $1, z$ cannot have neighbours in both components $G_{1}$ and $G_{2}$. We can assume that $z$ has a neighbour in $G_{1}$, the other case is treated similarly. Now, we look at possible neighbours of $z_{i}^{\prime}$ s, apart from, $y_{1}$. Note that by the above arguments, the only possible neighbours of $z_{i}$ in $T_{1}$ are $y_{1}, y_{2}, \ldots, y_{\delta-1}$ and $u$. If each $z_{i}$ is adjacent to $u$, then the set $\left\{w_{1}, w_{2}, \ldots, w_{\delta-1}, x_{2}, \ldots, x_{\delta-1}, y_{1}, y_{2}, \ldots, y_{\delta-1}, z, z_{1}, z_{2}, \ldots, z_{\delta-1}\right\}$ forms $4 \delta-4$ leaves of a tree in $G$, whose interior vertices are $u, x, y$ and $x_{1}$.

So, we can assume that at least one $z_{i}$, say $z_{1}$, is not adjacent to $u$. Since $z_{1}$ is adjacent to at most one vertex outside $T_{1}$ and $G$ is triangle-free, it follows by our choice of $u, x$ and $y$ that $z_{1}$ must be adjacent to $y_{1}, y_{2}, \ldots, y_{\delta-1}$ in $T_{1}$. Since $\operatorname{deg}\left(z_{1}\right) \geq \delta$, let $t_{1} \notin V\left(T_{1}\right)$ be a neighbour of $z_{1}$. Then $t_{1}$ has at least $\delta-1$ neighbours in $T_{1}$ which can only be $z_{1}, z_{2}, \ldots, z_{\delta-1}$, otherwise, we are done or we contradict our choice of $G$ as a triangle-free graph. Therefore, $t_{1}$ has a neighbour, say, $t_{2}$ not in $T_{1}$ which again must have at least $\delta-1$ neighbours in $T_{1}$, which must be $y_{1}, y_{2}, \ldots, y_{\delta-1}$. In particular, $t_{2}$ 
must be adjacent to $y_{1}$. Hence, the tree $T_{1} \cup\left\{y_{1} t_{2}\right\}$ has $4 \delta-4$ leaves and Lemma 1 holds. Therefore, in what follows, we assume that $u$ has no neighbour outside $T$.

Lemma 2 Let $A=\left\{w_{1}, w_{2}, \ldots, w_{\delta-1}\right\}$ and $B=\left\{z_{1}, z_{2}, \ldots, z_{\delta-1}\right\}$. If each element of $A$ or each element of $B$ is adjacent to $u$, then $G$ has a tree with at least $4 \delta-4$ leaves.

Proof of Lemma 2 Assume first that each element in $A \cup B$ is adjacent to $u$. Then the set $A \cup B \cup\left\{x_{1}, x_{2}, \ldots, x_{\delta-1}, y_{1}, y_{2}, \ldots, y_{\delta-1}\right\}$ forms $4 \delta-4$ leaves of a tree in $G$.

Consider the case where each element of $A$ is adjacent to $u$, the case for elements of $B$ can be treated similarly. Clearly, with all elements of $A$ attached to $u$ we have a tree with $4 \delta-5$ leaves and we are short of one leaf. We create that extra leaf as we did in the last part of the proof of Lemma 1 . That is, we consider possible neighbours of $z_{i}^{\prime} \mathrm{s}$, apart from $y_{1}$. Note that the only possible neighbours of $z_{i}$ in $T$ are $y_{1}, y_{2}, \ldots, y_{\delta-1}$ and $u$. Further, at least one $z_{i}$, say $z_{1}$, is not adjacent to $u$, otherwise, we are done, see previous paragraph. Now $z_{1}$ must be adjacent to $y_{1}, y_{2}, \ldots, y_{\delta-1}$, since $z_{1}$ has at most one neighbour outside $T$ and the aforementioned vertices are the only remaining possible neighbours of $z_{1}$ in $T$. Since $\operatorname{deg}\left(z_{1}\right) \geq \delta$, let $t_{1} \notin V(T)$ be a neighbour of $z_{1}$. Then $t_{1}$ has at least $\delta-1$ neighbours in $T$ which can only be $z_{1}, z_{2}, \ldots, z_{\delta-1}$, otherwise, we are done or we contradict our choice of $G$ as a triangle-free graph. Therefore, $t_{1}$ has a neighbour, say, $t_{2}$ not in $T$ which again must have at least $\delta-1$ neighbours in $T$, possibly, $y_{1}, y_{2}, \ldots, y_{\delta-1}$. In particular, $t_{2}$ must be adjacent to $y_{1}$. Hence, $t_{2}$ is an extra leaf gained and we are done. Therefore, Lemma 2 holds.

To complete the proof, it is adequate to consider the case where at least one element of $A$ is not adjacent to $u$ and at least one element of $B$ is not adjacent to $u$.

Lemma 3 Let $A$ and $B$ be as defined in Lemma 2. If at least one element of $A$ is not adjacent to $u$ and at least one element of $B$ is not adjacent to $u$, then $G$ has a tree with at least $4 \delta-4$ leaves.

Proof of Lemma 3 Assume without loss of generality that $w_{1}$ and $z_{1}$ are not adjacent to $u$. Consider first the case where there is a leaf of $T$ that is adjacent to at least two neighbours outside $T$. We may assume that $w_{1}$ has two neighbours outside $T$, say $r_{1}$ and $r_{2}$, other cases are treated likewise. Note that it cannot have at least three neighbours out, otherwise, we are done. Since $z_{1}$ is not adjacent to $u$ and cannot have two neighbours outside $T, z_{1}$ must be adjacent to $y_{1}, y_{2}, \ldots, y_{\delta-1}$. Since $\operatorname{deg}\left(z_{1}\right) \geq \delta$, let $t_{1} \notin V(T)$ be a neighbour of $z_{1}$. Then $t_{1}$ has at least $\delta-1$ neighbours in $T$ which can only be $z_{1}, z_{2}, \ldots, z_{\delta-1}$, otherwise, we are done or we contradict our choice of $G$ as a triangle-free graph. Therefore, $t_{1}$ has a neighbour, say, $t_{2}$ not in $T$ which again must have at least $\delta-1$ neighbours in $T$, namely, $y_{1}, y_{2}, \ldots, y_{\delta-1}$. In particular, $t_{2}$ must be adjacent to $y_{1}$. So, the tree $T \cup\left\{w_{1} r_{1}, w_{1} r_{2}, y_{1} t_{2}\right\}$ has $4 \delta-4$ leaves as desired. The same argument applies if there is a vertex of $G$ that has two neighbours outside $T$. So, we assume no vertex of $G$ has two neighbours outside $T$.

Consider the case where no vertex of $G$ has two neighbours outside $T$. We proceed as in the previous lemmas to show that there are vertices, $s_{2}$ and $t_{2}$ not in $T$ which are adjacent to $x_{1}$ and $y_{1}$, respectively. Then the tree $T \cup\left\{x_{1} s_{2}, y_{1} t_{2}\right\}$ has $4 \delta-4$ leaves and the result follows. 


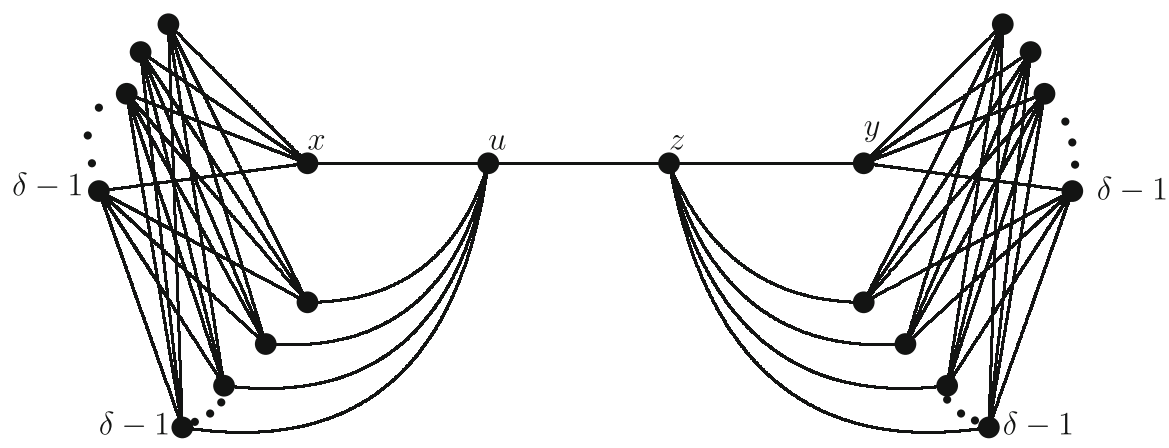

Fig. 2 The graph $G_{4 \delta}$

Consider possible neighbours of $z_{i}^{\prime}$ s, apart from, $y_{1}$. As before, the only possible neighbours of $z_{i}$ in $T$ are $y_{1}, y_{2}, \ldots, y_{\delta-1}$ and $u$. Then $z_{1}$ cannot have two neighbours out, otherwise, we are done. Since $z_{1}$ is not adjacent to $u$ and cannot have two neighbours outside $T, z_{1}$ must be adjacent to $y_{1}, y_{2}, \ldots, y_{\delta-1}$. Since $\operatorname{deg}\left(z_{1}\right) \geq \delta$, let $t_{1} \notin V(T)$ be a neighbour of $z_{1}$. Then $t_{1}$ has at least $\delta-1$ neighbours in $T$ which can only be $z_{1}, z_{2}, \ldots, z_{\delta-1}$, otherwise, we are done or we contradict our choice of $G$ as a triangle-free graph. Therefore, $t_{1}$ has a neighbour, say, $t_{2}$ not in $T$ which again must have at least $\delta-1$ neighbour in $T$, namely, $y_{1}, y_{2}, \ldots, y_{\delta-1}$. In particular, $t_{2}$ must be adjacent to $y_{1}$. By similar arguments, if we look at possible neighbours of $w_{i}^{\prime} \mathrm{s}$, apart from $x_{1}$, we end up having a vertex $s_{2} \notin V(T)$ which is adjacent to $x_{1}$. Hence, Lemma 3 is proven.

Thus, by following lemmas 1-3, all cases have been exhausted. Further, in each case whenever $u$ is a cut-vertex we obtain a tree with at least $4 \delta-4$ leaves as required. Therefore, the theorem holds.

To show that the bound on the leaf number in Theorem 4 is best in a sense, for each $\delta$, let $G_{4 \delta}$ be a graph Isomorphic to a graph formed by taking two complete $K_{\delta, \delta}$ bipartite graphs and join them by a bridge, say $e=u z$, see Fig. 2. Further, to see that Theorem 4 is sharp for both the bound on the order and the bound on the leaf number, let $G_{4 \delta-1}$ be a graph isomorphic to a graph formed from $G_{4 \delta}$ by collapsing the bridge $e=u z$ such that vertex $u$ becomes the same as vertex $z$, see Fig. 3 .

The following corollaries are consequences of Theorem 4.

Corollary 1 Let $G$ be a connected, triangle-free graph with minimum degree $\delta$ and leaf number $L(G)$ such that $L(G) \leq 4 \delta-5$. Then $G$ is 2-connected.

Corollary 2 Let $G$ be a connected, triangle-free graph with minimum degree $\delta$ and order $n$ such that $n \leq 4 \delta-2$. Then $G$ is 2-connected.

For graphs in general, the algorithm used to prove Theorem 4 can easily be employed to show that every simple, connected graph $G$ that has a cut-vertex has order $n \geq 2 \delta+1$ and the bound is tight as revealed by the family $G_{2 \delta+1}=K_{\delta}+K_{1}+K_{\delta}$.

Theorem 5 Let $G$ be a connected, triangle-free graph with minimum degree $\delta$ and order $n$ such that $n \leq 4 \delta-2$. Then $G$ is Hamiltonian or every longest cycle is a dominating cycle. 


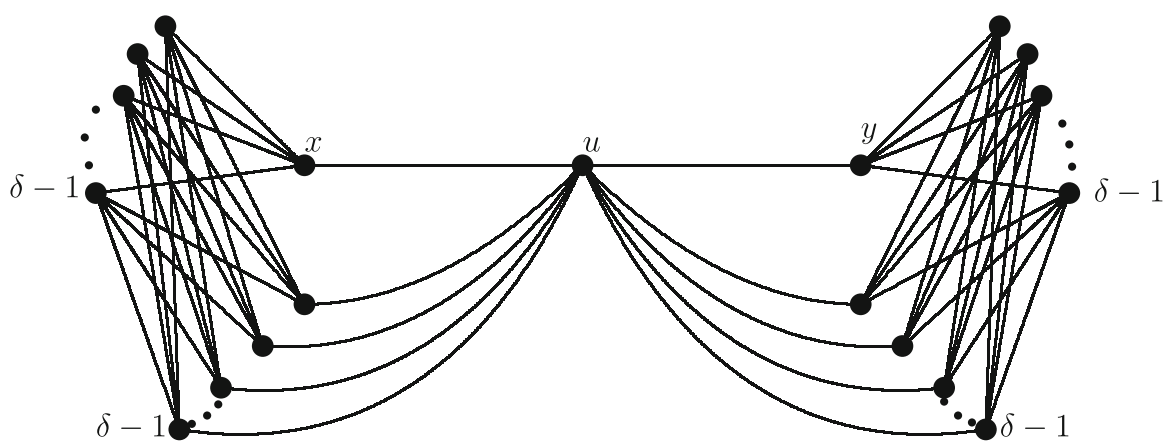

Fig. 3 The graph $G_{4 \delta-1}$

Proof By Corollary 2, $G$ is 2-connected. So, if $n \leq 4 \delta-3$, then the result follows by employing Theorem 2. Assume that $n=4 \delta-2$. Following Theorem 2, it is enough to consider the case where every longest cycle has length $4 \delta-4$ and where there are exactly two vertices outside the longest cycle. Let $C_{4 \delta-4}$ be a longest cycle in $G$ and $u_{1}, u_{2} \notin V\left(C_{4 \delta-4}\right)$ be distinct vertices not on $C_{4 \delta-4}$. To establish the result, assume to the contrary that $C_{4 \delta-4}$ is neither a spanning cycle of $G$ nor a dominating cycle. Then $u_{1} u_{2} \in E(G)$. The following arguments yield a contradiction showing that $u_{1} u_{2} \notin E(G)$ and the result is established.

We note first that for $i=1,2$ each $u_{i}$ has at least $\delta-1$ neighbours on $C_{4 \delta-4}$. Let $v_{t_{1}}, v_{t_{2}}, v_{t_{3}}, \ldots, v_{t_{\delta-1}} \in N\left(u_{1}\right)$ and $v_{s_{1}}, v_{s_{2}}, v_{s_{3}}, \ldots, v_{s_{\delta-1}} \in N\left(u_{2}\right)$ be neighbours of $u_{1}$ and $u_{2}$ on $C_{4 \delta-4}$, respectively. Since $G$ is a triangle-free graph and $u_{1} u_{2} \in E(G)$, $u_{1}$ cannot share a neighbour with $u_{2}$. Thus, the set

$$
A=\left\{v_{t_{1}}, v_{t_{2}}, v_{t_{3}}, \ldots, v_{t_{\delta-1}}, v_{s_{1}}, v_{s_{2}}, v_{s_{3}}, \ldots, v_{s_{\delta-1}}\right\}
$$

has $2 \delta-2$ distinct vertices of $C_{4 \delta-4}$. This implies that the set,

$$
B=\left\{v_{t_{1}+1}, v_{t_{2}+1}, v_{t_{3}+1}, \ldots, v_{t_{-1}+1}, v_{s_{1}+1}, v_{s_{2}+1}, v_{s_{3}+1}, \ldots, v_{s_{\delta-1}+1}\right\}
$$

has $2 \delta-2$ distinct vertices of $C_{4 \delta-4}$. Further, both $u_{1}$ and $u_{2}$ cannot have neighbours in $B$, otherwise, we obtain a cycle longer than $C_{4 \delta-4}$, which is not allowed. Thus, $A$ and $B$ are disjoint sets. Hence $V\left(C_{4 \delta-4}\right)=A \cup B$. The aforementioned arguments imply that $\operatorname{deg}\left(u_{1}\right)=\operatorname{deg}\left(u_{2}\right)=\delta$.

We claim first that no two consecutive vertices on $C_{4 \delta-4}$ are such that both of them are not in $A$. To prove this, assume contrary to the claim that there exist two consecutive vertices, say, $v_{i}$ and $v_{i+1}$ on $C_{4 \delta-4}$ such that $v_{i}, v_{i+1} \notin A$. Then $v_{i+1} \notin B$, since $v_{i} \notin A$. This means there is a vertex on $C_{4 \delta-4}$ which is neither in $A$ nor in $B$, a contradiction to $V\left(C_{4 \delta-4}\right)=A \cup B$. So, the first claim holds.

Secondly, we claim that whenever, $v_{t_{i}}$ is a neighbour of $u_{1}$ on $C_{4 \delta-4}$ then $v_{t_{i}+2} \notin$ $N\left(u_{2}\right)$. To prove this, suppose on the contrary that $v_{t_{i}+2} \in N\left(u_{2}\right)$. Then the cycle obtain from $C_{4 \delta-4}$ by deleting edges $v_{t_{i}} v_{t_{i}+1}, v_{t_{i}+1} v_{t_{i}+2}$ and replace them by edges $u_{1} v_{t_{i}}, u_{1} u_{2}, u_{2} v_{t_{i}+2}$ is longer than $C_{4 \delta-4}$, a contradiction. Therefore, the second claim is established. 
Lastly, we claim that for all $i, v_{t_{i}+2} \in N\left(u_{1}\right)$ whenever $v_{t_{i}} \in N\left(u_{1}\right)$. To settle this, assume to the contrary that $v_{t_{i}+2} \notin N\left(u_{1}\right)$ for some $i$. Then by previous arguments, $v_{t_{i}+1}$ and $v_{t_{i}+2}$ are to consecutive vertices not in $A$. This contradicts the first claim. Hence this claim holds.

The last claim implies that $\operatorname{deg}\left(u_{1}\right) \geq \delta+1$, a contradiction to the fact that $\operatorname{deg}\left(u_{1}\right)=$ $\delta$. Thus the assumption that $u_{1} u_{2} \in E(G)$ is false.

Therefore, $G$ must be Hamiltonian or every longest cycle is a dominating cycle in all cases.

Theorem 5 is best in the sense that each graph $G_{4 \delta-1}$ described before is a simple, connected, non-Hamiltonian, triangle-free graph in which every longest cycle is not dominating.

Corollary 3 Let $G$ be a connected, triangle-free graph with minimum degree $\delta$ and order $n$ such that $n \leq 4 \delta-1$. Then $G$ is traceable or every longest cycle is a dominating cycle.

Proof If $\delta=1$ then $n \leq 3$. Hence, $G$ is traceable for $\delta=1$.

Consider $\delta \geq 2$. Assume first that $G$ has a cut-vertex, say $u$. Let $u, x$ and $y$ be as defined in the proof of Theorem 4. By our choice of $u, x$ and $y$ together with the fact that $\delta$ is the minimum degree and $G$ is triangle-free, we obtain a tree isomorphic to $T$, using exactly the arguments we used to construct $T$ in the proof of Theorem 4 . So, it suffices to consider the tree to be $T$ as given in the proof of Theorem 4, see Fig. 1. Since $|V(T)|=4 \delta-1$ and $n \leq 4 \delta-1, T$ spans $G$. That is, $|V(G)|=|V(T)|$. Note that for all $i, j: 1 \leq j \leq \delta-1, p_{i}$ cannot be adjacent to $p_{j}$ for $p \in\{w, x, y, z\}$, since $G$ is triangle-free. This together with our choice of $u, x, y$ and the fact that $G$ is triangle-free imply that for each $i$, the $\delta$ neighbours of $z_{i}$ must be $u, y_{1}, y_{2}, \cdots, y_{\delta-1}$. Similarly, for all $i$, the $\delta$ neighbours of $w_{i}$ must be $u, x_{1}, x_{2}, \cdots, x_{\delta-1}$. Therefore, $G=G_{4 \delta-1}$ and is traceable.

Consider the case where $G$ is 2-connected. If $n \leq 4 \delta-2$ then the result is an immediate consequence of Theorem 5. Assume that $n=4 \delta-1$. Following Theorem 2 , it is adequate to consider the case where every longest cycle $C$ is of length $4 \delta-4$ and $G-C=K_{2} \cup K_{1}$, otherwise, we are done easily. Let $u_{1}, u_{2}, u_{3} \notin V(C)$. We may assume that $u_{1} u_{2} \in E(G)$ and $u_{1} u_{3}, u_{2} u_{3} \notin E(G)$, other cases are treated likewise. Since $u_{1} u_{2} \in E(G)$ and $|V(C)|=4 \delta-4$, one can easily use similar arguments as the proof of Theorem 5 for $n=4 \delta-2$ to get a contradiction. Hence the result follows.

We now improve Theorem 3 by proving the following result:

Theorem 6 Let $G$ be a connected, claw-free, paw-free graph. Then $G$ is Hamiltonian if and only if $G$ is not a path.

Proof Assume first that $G$ is a tree. Then $G$ is a path, otherwise, $G$ contains a claw, which is not allowed. So, assume that $G$ is not a tree. Then $G$ contains at least one cycle. Let $C_{k}=v_{1}, v_{2}, v_{3}, \ldots, v_{k}, v_{1}$ be a longest cycle in $G$. It suffices to show that $C_{k}$ spans $G$. Assume contrary to the claim that $C_{k}$ does not span $G$. Then, since $G$ is connected there is a vertex, say, $v$ which is not on $C_{k}$ and $v$ is adjacent to a vertex, 
say, $v_{i} \in V\left(C_{k}\right)$. Now, $v$ cannot be adjacent to $v_{i-1}$ or $v_{i+1}$, otherwise, we contradict our choice of $C_{k}$ as a longest cycle in $G$. Thus, if $v_{i-1}$ is not adjacent to $v_{i+1}$, then the set $\left\{v, v_{i-1}, v_{i}, v_{i+1}\right\}$ forms a claw, which is prohibited. If on the other hand, $v_{i-1}$ is adjacent to $v_{i+1}$, then the set $\left\{v, v_{i-1}, v_{i}, v_{i+1}\right\}$ yields a paw in $G$, which is not permitted. Hence, in either case we get a contradiction. So the supposition that $C_{k}$ does not span $G$ is false. Consequently, $G$ is Hamiltonian.

From Theorem 6, one can easily deduce that every simple, connected, claw-free, paw-free graph is traceable.

\section{Conclusion}

Sufficient conditions for a connected, triangle-free graph to be 2-connected based on minimum degree and leaf number or minimum degree and order have been presented. The results are crucial in the establishment of sufficient conditions for a trianglefree graph to be Hamiltonian. In fact, the results have been used to deduce sufficient conditions for the existence of spanning paths or cycles in triangle-free graphs. The algorithm used to establish sufficient conditions for a triangle-free graph to be 2connected can also be used for $C_{4}$-free graphs and graphs of given girth. It would be interesting if sharp or asymptotically tight conditions on 2-connectedness can be obtained for $C_{4}$-free graphs or graphs of given girth based on the aforementioned parameters.

\section{Declarations}

Conflict of interest The authors have no conflicts of interest to declare that are relevant to the content of this article.

\section{References}

1. Aung, M.: Longest cycles in triangle-free graphs. J. Combin. Theory Ser. B 47(2), 171-186 (1989)

2. Bauer, D., Kahl, N., McGuire, L., Schmeichel, E.: Long cycles in 2-connected triangle-free graphs. Ars Comb. 86, 295-304 (2008)

3. Brandt, S.: Cycles and paths in triangle-free graphs. In: The mathematics of Paul Erdős, II, volume 14 of Algorithms Combin, pp. 32-42. Springer, Berlin (1997)

4. Broersma, H.J., van den Heuvel, J., Veldman, H.J.: A generalisation of Ore's theorem involving neighbourhoods unions. Discrete Math. 122, 37-49 (1993)

5. Dirac, G.A.: Some theorems on abstract graphs. Proc. Lond. Math. Soc. 2, 69-81 (1952)

6. Duffus, D., Gould, R.J., Jacobson, M.S.: Forbidden subgraphs and Hamiltonian theme. In: Chatrand, G., et al. (eds.) The theory and applications of graphs: fourth international conference, pp. 297-316. Wiley, New York (1981)

7. Fernandes, L.M., Gouveia, L.: Minimal spanning trees with a constraint on the number of leaves. Eur. J. Oper. Res. 1104, 250-261 (1998)

8. Garey, M.R., Johnson, D.S.: Computers and intractability: a guide to the theory of NP-completeness. W.H. Freeman and Company, San Francisco (1979)

9. Goodman, S., Hedetniemi, S.: Sufficient conditions for a graph to be Hamiltonian. J. Comb. Theory B 16, 175-180 (1979)

10. Harris, J.M., Mossinghoff, M.J.: Traceability in small claw-free graphs. Util. Math. 70, 263-271 (2006)

11. Mafuta, P.: Leaf number and Hamiltonian $C_{4}$-free graphs. Afr. Mat. (2017). https://doi.org/10.1007/ s13370-017-0503-y 
12. Mafuta, P., Mukwembi, S.: On minimum degree, leaf number, traceability and Hamiltonicity in graphs. Discrete Appl. Math. (2017). https://doi.org/10.1016/j.dam.2016.12.028

13. Mafuta, P., Mukwembi, S., Munyira, S.: Spanning paths in graphs. Discrete Appl. Math. (2018). https:// doi.org/10.1016/j.dam.2018.08.001

14. Mafuta, P., Mukwembi, S., Munyira, S., Vetrík, T.: Hamiltonicity, leaf number and minimum degree. Acta Math. Hung. 152(1), 217-226 (2017)

15. Mafuta, P., Mushanyu, J.: Spanning paths and cycles in triangle-free graphs. Queastiones Math. (2019). https://doi.org/10.2989/16073606.2019.1654005

16. Mukwembi, S.: Minimum degree, leaf number and traceability. CMJ 63, 539-545 (2013)

17. Mukwembi, S.: On spanning cycles, paths and trees. Discrete Appl. Math. 161, 2217-2222 (2013)

18. Nash Williams, C.: Edge disjoint Hamiltonian circuits in graphs with vertices of high valency. In: Studies in pure mathematics, pp. 157-183. Academic Press, Cambridge (1971)

19. Ore, O.: A note on Hamiltonian circuits. Amer. Math. Monthly 67, 55 (1960)

20. Stojmenović, I.: Topological properties of interconnection networks. In: Cheng, M.X., Li, Y., Du, D. (eds.) Combinatorial optimization in communication networks, pp. 427-466. Springer, Boston (2006)

Publisher's Note Springer Nature remains neutral with regard to jurisdictional claims in published maps and institutional affiliations. 